\title{
A Hierarchical Prediction Method Based on Hybrid- kernel GWO-SVM For Metal Tube Bending Wrinkling Detection
}

\section{Shuyou Zhang}

Zhejiang University

\section{Yujun Yuan}

Zhejiang University

zili wang ( $\square$ ziliwang@zju.edu.cn )

Zhejiang University https://orcid.org/0000-0002-4765-4410

Yaochen Lin

Zhejiang University

Lanfang Jiang

Zhejiang University

Mengyu Fu

Zhejiang University

\section{Research Article}

Keywords: Metal tube bending, Wrinkling, Hierarchical prediction method, Hybrid-kernel, GWO-SVM

Posted Date: January 20th, 2022

DOI: https://doi.org/10.21203/rs.3.rs-1258909/v1

License: (1) This work is licensed under a Creative Commons Attribution 4.0 International License.

Read Full License 


\title{
A hierarchical prediction method based on hybrid-kernel GWO-SVM for
}

\section{metal tube bending wrinkling detection}

\author{
Shuyou Zhang ${ }^{1,2}$, Yujun Yuan ${ }^{1}$, Zili Wang ${ }^{1,2 *}$, Yaochen $\operatorname{Lin}^{3}$, Lanfang Jiang ${ }^{4}$, Mengyu Fu ${ }^{1}$ \\ (1. State Key Laboratory of Fluid Power and Mechatronic Systems, Zhejiang University, Hangzhou, 310027, China. 2. Engineering Research Center for Design \\ Engineering and Digital Twin of Zhejiang Province, Zhejiang University, Hangzhou, 310027, China 3. King-Mazon Co., Ltd., Lishui, 323000, China. 4. Zhijiang \\ College of Zhejiang University of Technology, Shaoxing 312030, China) \\ *Corresponding author: Zili Wang, e-mail:ziliwang@zju.edu.cn.
}

\begin{abstract}
Metal bending tube is widely used in industry while its forming defects extremely affect the bending quality. Among all defects, the bending-inside wrinkling caused by the non-uniform compressive stress is a zero-tolerated defect, particularly when the tube is for transportation. However, the current wrinkling detection approach, suffering from the lack of insight into wrinkling mechanism, is normally posteriori. To obtain the priori wrinkling condition for a certain go-to-bend tube, we put forward a metal tube bending wrinkling hierarchical prediction method based on hybrid-kernel Gray Wolf Optimizer (GWO) Support Vector Machine (SVM). Three typical kernel combinations are utilized for the GWO-SVM prediction model. To verify the proposed wrinkling prediction method, aluminum alloy series tubes are tested. By constructing the 12 typical designations aluminum alloy tubes' finite element bending simulation case base, the prediction model is trained through three hybrid-kernel GWO-SVMs, respectively. The results are compared with the traditional SVM and GWO-SVM, which show that the proposed hybrid-kernel GWO SVM model has the best performance for hierarchically predicting bending wrinkling. This proposed prediction method lays the foundation for metal tube bending wrinkling detection.
\end{abstract}

Keywords: Metal tube bending; Wrinkling; Hierarchical prediction method; Hybrid-kernel; GWO-SVM

\section{Introduction}

With a hollow structure and good mechanical properties, different shapes of metal bending tubes are usually used for equipment lightweight, liquid transportation, and anti-collision buffer [1]. Under the combined action of one side tension and one side pressure, the tube blank is formed into a bending tube [2]. Due to the complex deformation conditions, it will inevitably produce diverse forming defects, such as bending springback [3], wall thickness thinning [4], cross-section distortion [5], wrinkling [6], etc.

The tube bending deformation defects can be generally categorized into axial defects and radial defects. Axial defects, mainly caused by unloading springback and elongation, will directly affect the tube assembly performance in service. Springback refers to the actual bending angle that becomes lower than the set bending angle [7]. The bending tube produces a certain amount of springback angle along the axial direction, which is due to the recovery of elastic deformation of the tube [8]. The yield strength and elastic modulus of the tube material are important factors that affect the springback angle [9]. At the same time, radial defects are distributed along the diameter of the tube. The thinning of the tube wall is caused by the stretching of the tube after receiving the tangential tensile stress in the process of bending. The elongation length and the relative bending radius have a great influence on the wall thickness reduction during the forming process. The smaller the relative bending radius and the larger the elongation, the more serious the thinning of the wall thickness [10]. The flattening of a cross-section is also a defect in the radial direction, which is mainly reflected by the flattening of the outer side of the tube. The cross-section flattening is mainly affected by the material of the tube. The contraction strain ratio and the elastic modulus of the tube will change the cross-section flattening. Furthermore, the bending angle is an important factor affecting the flattening of the section [11]. Unlike the above-mentioned defects, the wrinkling on the tube bending inner side occurs both axially and radially. During the tube bending process, the wrinkling wave is formed in the axial direction with large deformation along the bending direction, while each cross-section of the bent tube also shows varying depression deformation in the radial direction.

When the compressive stress exceeds a certain critical value, the local plastic buckling instability will occur, resulting in 
wrinkling. When this compression occurs continuously in a certain area, the excessively accumulated local material is difficult to spread in time, finally resulting in wrinkling. The wrinkling of a bending tube is mainly studied from two aspects. A part of researchers focused on theoretical studies of tube bending wrinkling mechanism. To effectively predict the appearance of wrinkle defects during the forming process, Zhao et al. [12] used strain diagrams to evaluate the formation process of external pressure and analyzed the influence of geometric parameters on elastoplastic instability. Yang et al. [13] established a wrinkle prediction model to predict the minimum radius of tube bending and analyzed the influence of process parameters such as bending angle, tube geometry, and material properties on the forming quality, based on the thin shell theory, forming theory, energy principle and wave function. In addition, they also studied the potential effect of friction on the bending deformation of thin-walled tubes with small bending diameters. The dynamic contact condition of the bending process with large slip was reproduced by the torsion compression test, the results showed the lower sensitivity of wrinkling to friction [14]. The other part of the researchers concentrated on the influential factors study of wrinkling by numerical simulation. Cui et al. [15] studied the influence of process parameters on the wrinkling of hydro-formed thin-walled tubes and obtained the influence of elastic modulus, initial yield stress, and tangent modulus on the tube wrinkling through the finite element method. Hasanpour et al. [16] quantitatively studied the wrinkling phenomenon of the thin-walled tube in rotary bending and the effect of some process parameters by detecting wrinkling in a finite element model using velocity integral parameters. Furthermore, Alexander et al. [17] took the geometric shape of wrinkles $\mathrm{h} / \mathrm{L}$ as a geometric feature to describe the severity of wrinkles and obtained the Stress Concentration Factor (SCF) related to wrinkles through finite element analysis to determine the failure period. Li et al. [18] presented an analytical method for obtaining the wrinkling limit of thin-walled tubes by finite element simulation and formulating reasonable analytical assumptions.

Since the mechanism of metal forming wrinkling defects is extremely complex, it is difficult to theoretically obtain the wrinkling information according to the forming conditions. Recently, intelligent algorithms are widely applied in many metal forming defects problems ranging from the prediction of the occurrence of defects in the sheet metal forming process [19], the control of the springback phenomenon in the aluminum sheet bending process [21], and realize real-time control of the rebound of the sheet [20]. SVM method focuses on solving classification and regression problems in engineering, which also better solve practical problems such as small samples, nonlinearity, high dimensionality, and local minima [22]. But for uneven data and large-scale problems, general shortcomings such as complexity of algorithms, low efficiency, and low classification performance still exist in SVM methods. Generally, SVM uses a kernel function to map origin input data into a high dimensional feature space and finds a hyperplane determined by maximizing the gap between classes to classify data. In the process of finding optimal hyperplane, regularization parameter $(\mathrm{C})$ determines the tolerance of error while the kernel parameter (g) implicitly determines the distribution of the data after it is mapped to a new feature space. To find the appropriate parameters for better achieving the classification purpose, SVM integrated with various optimization algorithms are proposed to improve the forecasting ability, such as Particle Swarm Optimizer (PSO) [23] and Genetic Algorithm (GA) [24]. Due to the GWO assisted with encircling mechanism can find the best solutions which can be extended to higher dimensions as a hyper-sphere, it shows superior performance in terms of optimizing parameters [25]. Based on the safety diagnosis of automated vehicles, Shi et al. [26] proposed an under-sampling method based on linear discriminant analysis and a threshold adjustment method based on the GWO algorithm to improve the performance of SVM model classification and fault diagnosis. $\mathrm{Xu}$ et al. [27] constructed the demand forecasting model using the SVM based on GWO. The results show that the GWOSVM model obtained a better result than that of the BP neural network model and SVM model.

For most of the tube practical applications, the wrinkling defects are zero-tolerated. Suffering from the lack of insight into wrinkling mechanisms, the existing methods are normally posteriori. To aforehand obtain the priori wrinkling condition for a certain go-to-bend tube blank, a metal tube bending wrinkling hierarchical prediction method based on the novel hybridkernel GWO-SVM was proposed. By finite element analysis of the tube bending process, a wrinkling hierarchical method is obtained to determine the degree of wrinkling. To obtain a more accurate prediction model, a novel hybrid-kernel GWO-SVM is established. A case study is carried out by a series of aluminum alloy tubes to verify the feasibility of the proposed method. In addition, an experiment based on the 6063-aluminum tube bending is carried out. The results show that the novel hybridkernel GWO-SVM method is a proper model for hierarchically predicting metal tube bending wrinkling detection

The rest of this paper is organized as follows. Sect.2 described the bending wrinkling problem. Then, the wrinkling hierarchical prediction method using the Hybrid-kernel GWO-SVM algorithm was presented in Sect.3. To verify the prediction method, an aluminum alloy tube bending case was conducted. The results of different wrinkling prediction models 
were compared and discussed. Finally, some main conclusions were drawn in Sect. 5.

\section{Bending Wrinkling Problem Statements}

Rotary Draw Bending (RDB) is the most commonly used metal tube bending form. The main components of a typical RDB equipment are shown in Fig. 1, which combined with the bending die, insert, clamp die, wiper die, and pressure die. The bending die rotates about the X-direction while the tube blank is fitted in its groove. With the exact same dimensions with the bending die, the insert provides the tension required for tube bending through the contact friction along with the clamp die. The wiper die enhances the support area evenly along the bending inner side against wrinkling. The pressure die provides boost in the Z-direction force that helps to restrain the material flow in the bending outer side. The wrinkling of tube bending is the result of the interaction of various deformation conditions. In the process of bending, the concave side of tube wall shrinks along the bending direction due to the tangential compressive stress, which will inevitably cause the increase in tube wall thickness while the excessive increase will cause wrinkling as is shown in Fig.1. The wall thickness distribution is influenced by relative bending radius $(R / D)$, relative wall thickness $(t / D)$, and the tube diameter $(D)$. The smaller $t / D$ and $R /$ $D$, the more the tangential compressive stress on the concave side of the tube wall, which leads to a more serious wall thickness increase and the possibility of the occurrence of wrinkling. The speed of the bending die $(\omega)$ and the bending radius $(r)$ can also affect the wrinkling. Hence, whether the relative speed $(d V p)$ between the boosting speed of the pressure die and the speed of the bending die is greater than zero represents the direction of the friction force acting on the outside of the tube. Large frictional resistance with high friction factor between tube and die $(\mu)$ causes tangential tension on the outer convex side of the tube wall, which relieves the tangential compression and material accumulation of the inner concave side of the tube wall to some extent, thereby affecting the wrinkling tendency of the inner concave side of the tube. Be considered to place the tube into the bending die, the gap $\left(t_{0}\right)$ between the tube wall and the die is unavoidable. Yet the gap will influence the material flow space which will also influence the bending wrinkling. Though the pressure die can reduce the thinning of the outer side of the tube wall if it boosts for a whole forming process, it can also cause the thickness of the inner concave sidewall and even cause wrinkling. Thus, the initial position $\left(L p_{0}\right)$ is the factor affecting wrinkling. Moreover, as the wiper die has the ability to support bending the inner area against wrinkling, the length of the wiper die $(L)$ has a great influence on the wrinkling while the bending angel determines whether the wrinkling could show up. In addition to the effect of process parameters on wrinkling detection, the material of the tube also makes a significant contribution to affect wrinkling since each tube with different parameters of the initial yield stress $\left(\sigma_{s 0}\right)$ and Young's modulus $(E)$ strongly affect the wrinkling ability of the tube materials has different strength, toughness, and mechanical properties. Accordingly, the effect factors of metal tube bending wrinkling $(X)$ are consistent with the mentioned parameters, as shown in Eq. (1).

$$
X=\left\{R / D, D, t / D, \mu, t, d V p, L p_{0}, \omega, L, r, E, \sigma_{s 0}\right\}
$$

The wrinkle waviness of a bent tube is usually used as a criterion for judging the wrinkling degree. The formula for calculating the waviness is shown in Eq. (2).

$$
\Delta H=\frac{D_{1}-D_{2}}{2}
$$

Where $D_{l}$ denotes the maximum diameter in the direction of the short axis of the waving curve, while $D_{2}$ denotes the minimum diameter in the direction of the short axis of the waving curve.

The evaluation of the wrinkling of a bent tube can be performed by first layering the tube along the $X$-axis and then calculating the wrinkle waviness of each layer on the $\mathrm{Y}-\mathrm{Z}$ plane. The center layer where $\mathrm{X}=0$ is defined as the zero layers, while other layers are defined as the $m$ th layer according to the absolute value of $Z$ from small to large and whether it is placed above the center layer or below it, for example, $X=3, m=1 ; X=-3, m=2 ; X=5, m=3 \ldots$ and so on. On the $m$ th layer, the distance between the point on the inner side of a tube wall and the bending center is calculated. As shown in Fig.2, the red point $N_{i}^{m}\left(x_{i}^{m}, y_{i}^{m}, z_{i}^{m}\right)$ represents the $i$ th point which has the maximum distance on the $m$ th layer, while the blue point 
$C_{j}^{m}\left(X_{j}^{m}, Y_{j}^{m}, Z_{j}^{m}\right)$ represents the $j$ th minimum points on the $m$ th layer. $O(0,0,0)$ represents the center of the bending. Then, the minimum distance $D_{j}^{m}$ and maximum distances $L_{i}^{m}$ are calculated in Eq. (3).

$$
\begin{aligned}
& D_{j}^{m}=\sqrt{X_{j}^{m 2}+Y_{j}^{m 2}+Z_{j}^{m 2}} \\
& L_{i}^{m}=\sqrt{x_{i}^{m 2}+y_{i}^{m 2}+z_{i}^{m 2}}
\end{aligned}
$$

Then, the wrinkling waviness can be obtained in Eq. (4).

$$
H_{m}(i, j)=L_{i}^{m}-\frac{D_{j}^{m}+D_{j+1}^{m}}{2}
$$

The tube bending wrinkling waviness of a bent tube on the $m$ th layer is shown in Fig. 2 .

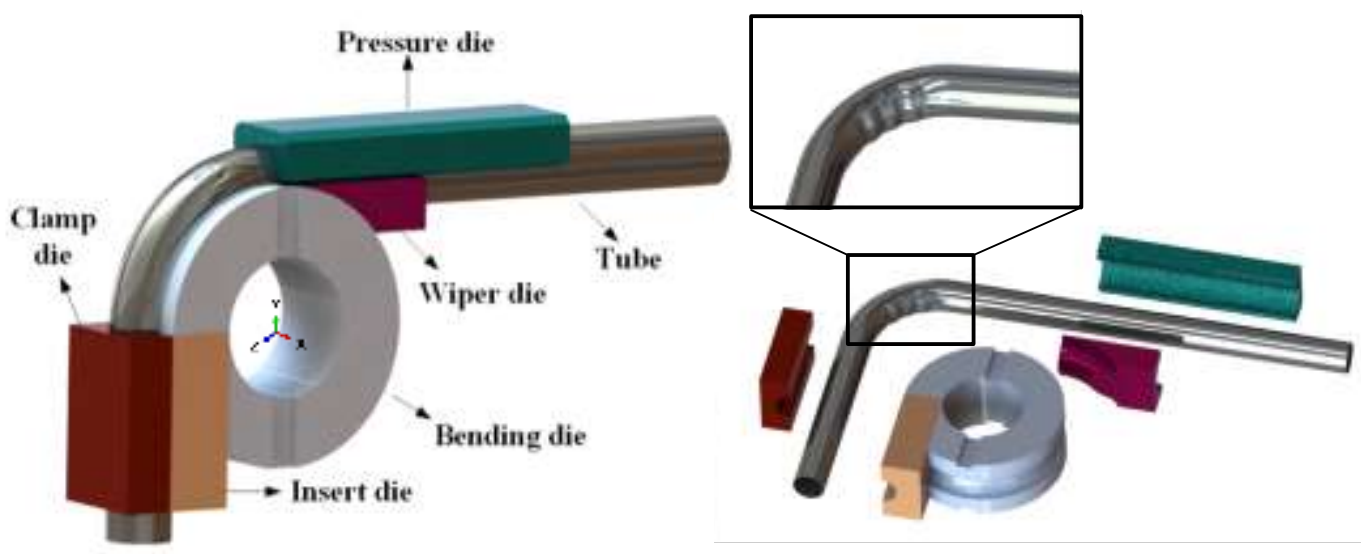

Fig. 1 RDB components and bending wrinkling diagram
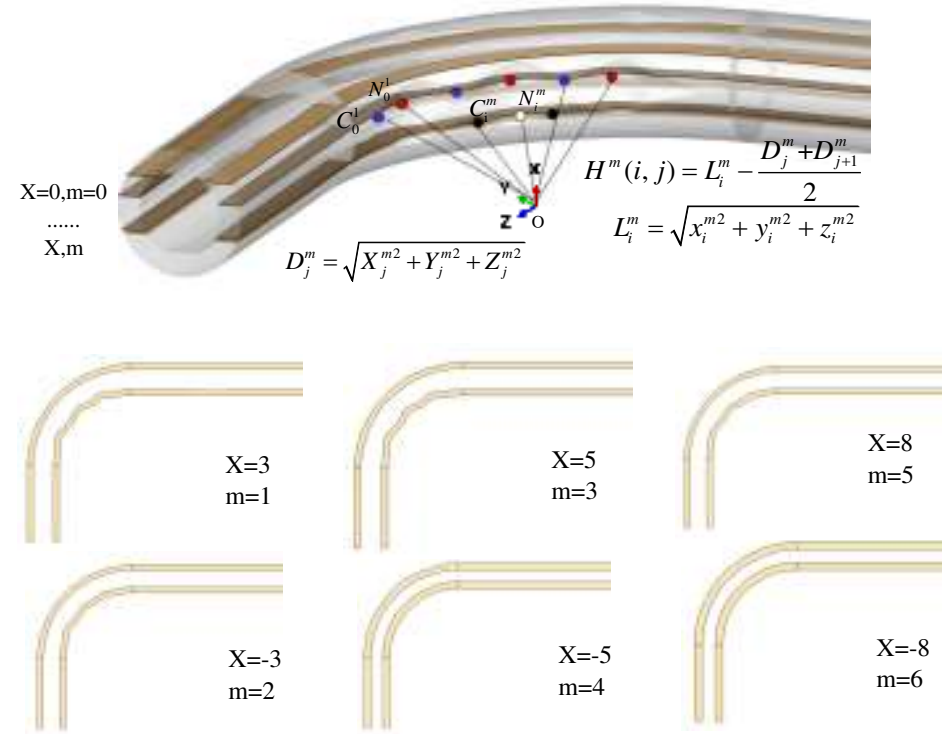

Fig. 2 Tube bending wrinkling waviness multi-layer extraction 


\section{Wrinkling prediction approach based on Hybrid-kernel GWO-SVM}

\subsection{Construction of wrinkling database}

To obtain the priori wrinkling condition for a certain go-to-bend tube, a database of supervised training samples need to be established. The input data are the material parameters and process parameters that affect the wrinkling of metal tube bending, as shown in Eq. (1). By the given range, the parameters can be obtained by Latin hypercube sampling. With the aim of rapidly obtaining a large scale of wrinkling samples, the tube bending models are established by simulating method using finite element simulation software, such as ABAQUS/Explicit.

As well as determining the input variables, the samples also need to be labeled for supervised training. Yet even the tube has been simulated, the precise wrinkling information cannot be obtained, including the judgment of slight wrinkling, the number of wrinkling, and the degree of wrinkling. Therefore, further processing of the finite element model is needed for getting complete wrinkle information. In the process of finite element numerical calculation, it is necessary to mesh the complex model, discretize the mass of a continuum element, and solve the equations at each element node. For the tube bending model, the element nodes on the tube which are used for numerical calculation are extracted and imported into MATLAB. Subsequently, the corresponding position data of each element node before and after the bending are extracted. As shown in Fig. 2, the node positions on each layer of the Y-Z plane on the outside of the tube are obtained as well as the wrinkling waviness can be calculated in Eq. (4) and Eq. (5).

$$
L=\sqrt{y^{2}+z^{2}}
$$

$L$ represents the distances between each node on the Y-Z plane and the center point of the bending die. The points where $L^{\prime}=0$ and $L^{\prime \prime}>0$ are set as $N_{i}$, which denotes the $i$ th point which has the maximum $L$, while the points where $L^{\prime}=0$ and $L^{\prime \prime}<0$ are set as $C_{j}$, which denotes the $j$ th point which has the minimum $L . h_{i}$ represents the depth of each wrinkle is obtained in Eq. (5).

$$
N_{i} \in\left\{\left(x_{i}, y_{i}\right)\right\}, C_{j} \in\left\{\left(x_{j}, y_{j}\right)\right\}, h_{i}=\left|y_{i}-\right| \frac{y_{j}-y_{j+1}}{2}||
$$

Considering that there are some interference points when extracting the element nodes, these points will be eliminated according to the ratio of wrinkle depth and tube diameter $(h / D)$. Moreover, the slight wrinkling can also be ignored. The wrinkles where $h / D$ is larger than $1 / 50$ and the ratio of the difference between the vertical coordinates of the maximum point and the minimum point on the left and right sides and $D$ is larger than 1/100 remain. Then, the angle between the line connecting the two sides of the wrinkles and the origin (calculated by vector method), representing the angle of each wrinkle $(j)$, is taken. The wrinkle depth matrix $(H)$ and the matrix of angle $(\mathrm{J})$ are the average value of $h$ and $j$ on the $m$ th layer, respectively. Based on the appearance of wrinkling and the theory of waviness, it is easy to draw the conclusion that the bigger $H$ and the smaller $J$, the higher the wrinkle degree. As a result, the depth-to-width ratio of wrinkles $(P)$ as the criterion to measure the wrinkle degree is calculated in Eq. (6).

$$
P=H / J
$$

Define the wrinkle criterion: where $H / D$ is larger than $1 / 50$ and the difference between the maximum point and the minimum point on the left and right sides are larger than 1/100 means there's a wrinkle at that point. For further estimating the tube bending wrinkling, both wrinkling numbers $(n)$, which is the dimension of the $H$ matrix, and $P$ are considered to establish. The hierarchical classification method is proposed in Fig. 3. 


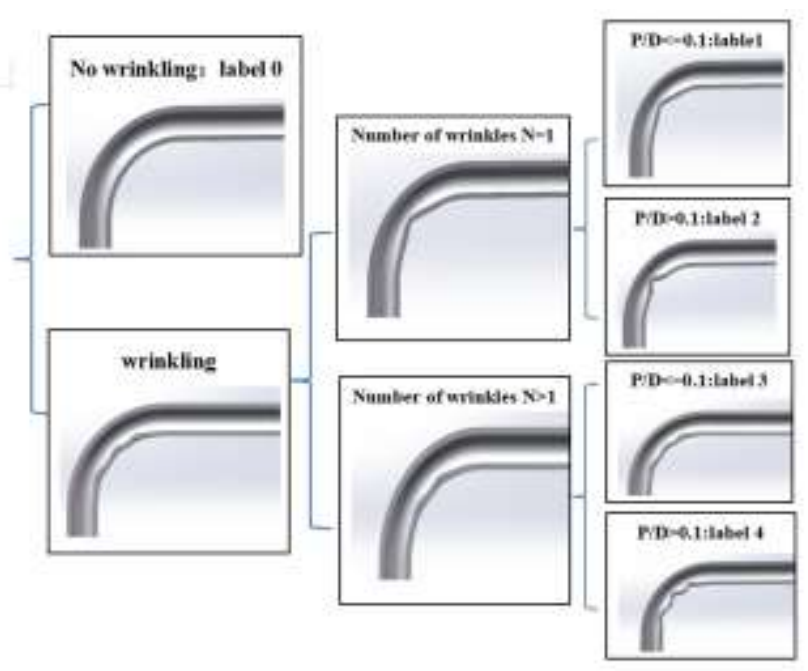

Fig. 3 Tube wrinkling hierarchical labels

\subsection{Hybrid-kernel GWO-SVM prediction model}

Based on the above studies, we established the input matrix $X$ in Eq. (1), and the label $Y$ is also determined based on the hierarchical classification method as shown in Eq. (7).

$$
Y \Leftrightarrow\{P, n\}
$$

For realizing the prediction of bending tube winkling, it is needed to establish the mapping between $\mathrm{X}$ and $\mathrm{Y}$, which can be achieved through algorithms. Machine learning algorithms could find the relationship between input and output samples by learning a large amount of samples. SVM method focuses on solving classification problems, which also comes powerful tools to overcome some traditional difficulties such as the "curse of dimensionality", "over-fitting" etc. Error! Reference source not found. But for uneven data and large-scale problems, general shortcomings such as complexity of algorithms, low efficiency and low classification performance Error! Reference source not found. still exist in SVM methods. To reach higher accuracy and efficiency of hierarchical predicting of tube bending wrinkling, we optimized the traditional SVM and proposed a novel hybrid-kernel GWO-SVM method to establish the wrinkling prediction model.

\section{Basic SVM}

The original SVM is based on the given training sets to find a hyperplane in the sample space and separate the different samples Error! Reference source not found.

$$
D \in\left\{\left(x_{j}, y_{j}\right)\right\}, x_{i} \in R^{n}, y \in\{-1,+1\}
$$

The partition hyperplane can be expressed by a linear equation in Eq. (9), where $b$ is the normal vector of the hyperplane, representing the direction of the hyperplane

$$
w^{T} x+b=0, w=\left(w_{1}, w_{2}, \ldots, w_{d}\right)
$$

To find the optimal hyperplane which can be better used for classification, the distance between the vector closest to the hyperplane and the hyperplane is maximized. The basic form can be expressed as Eq. (10).

$$
\begin{aligned}
& \min \frac{1}{2}\|w\|^{2} \\
& \text { s.t. } y_{i}\left(w^{T} x_{i}+b\right) \geq 1, i=1,2, \ldots m
\end{aligned}
$$

The solution can also be written in Lagrange form as Eq. (11).

$$
L(w, b, \alpha)=\frac{1}{2}\|w\|^{2}+\sum_{i=1}^{m} \alpha_{i}\left(1-y_{i}\left(w^{T} x_{i}+b\right)\right)
$$

To obtain the dual problem, the partial derivatives of $W$ and $B$ are set to 0 , as Eq. (12). 


$$
\begin{aligned}
& \max \sum_{i=1}^{m} \alpha_{i}-\frac{1}{2} \sum_{i=1}^{m} \sum_{j=1}^{m} \alpha_{i} \alpha_{j} y_{i} y_{j} x_{i}^{T} x_{j} \\
& \text { s.t. } \sum_{i=1}^{m} \alpha_{i} y_{i}=0, \\
& \alpha_{i} \geq 0, i=1,2, \ldots, m
\end{aligned}
$$

Instead of directly calculating the inner product of high dimensional or even infinite-dimensional characteristic space, consider a 'kernel function' such as Eq. (13):

$$
\kappa\left(x_{i}, x_{j}\right) \leq \varphi\left(x_{i}\right), \varphi\left(x_{j}\right) \geq \varphi\left(x_{i}\right)^{T} \varphi\left(x_{j}\right)
$$

$\varphi\left(x_{i}\right)^{T} \varphi\left(x_{j}\right)$ is the input vector to the feature space mapping Error! Reference source not found.

In the SVM algorithm, $C$ is the so-called 'regularization parameter' that controls the trade-off between empirical error and complexity of the hypothesis space used. If $C$ is too large, it will tend to use empirical data and ignore the complexity of the structure, otherwise, it will make the structure too complex and ignore the role of empirical data. Gamma $(g)$ denotes a kernel parameter, which implicitly determines the distribution of the data after it is mapped to a new feature space. In traditional SVM algorithms, $C$ and $g$ are obtained through grid optimization and cross-validation. Besides, the choice of kernel function has a great influence on the prediction result. Different conventional kernels have different technical merits in classification. Commonly used kernel functions include linear kernel, polynomial kernel, RBF kernel, and Sigmoid kernel.

\section{GWO-SVM}

GWO is an intelligent optimization algorithm based on the gray wolf's predation. It has been applied to parameter optimization, job shop scheduling, and so on. The GWO process divides the gray wolf into four categories according to the social dominant level, namely $\alpha, \beta 、 \gamma$ and $\omega$. These four classes represent higher management capability from top to bottom and the highto-low fitness solutions in GWO. The wolf's hunting process involves closing in on the prey, taking the three positions closest to the prey in each iteration, and surrounding the prey 32. In this paper, we optimized the traditional SVM model using GWO to find the best parameters in SVM. The steps of GWO-optimized SVM parameter selection are as followed.

Step 1 Initialize Gray Wolf population, the number of iterations, and SVM parameters $C$ and $g$.

Step 2 Calculate fitness, taking the minimum error rate of SVM as the fitness value:

$$
\text { Fitness }=100-\text { accuracy }
$$

Step 3 The fitness values are compared and three optimal solutions are obtained.

Step 4 Update individual gray wolf location

Step 5 According to the Algorithm to determine whether the maximum number of iterations, if yes then stop, if no then continue to repeat the operation.

Step 6 save the best $C$ and $g$ by the final location of the $\alpha$ wolf

Step 7 Train SVM model and predict the test samples

\section{Hybrid-kernel strategy}

Each kernel function is suitable for some tasks, and it must be chosen for the tasks under consideration by hand or using prior knowledge. It is found that different kernel functions have different technical merits in classification and show different prediction performance when other parameters are the same. For example, the linear kernel has the advantage of simpler parameters and faster computation compared to other kernels. Furthermore, the RBF kernel, as a local kernel function, has strong learning ability as it maps samples to a higher-order hyperplane to achieve nonlinear mapping while it has weak generalization performance. On the contrary, the polynomial kernel, as a global kernel function, has strong generalization ability is strong and weak learning ability 33 . Therefore, by combining conventional kernel functions, the combined feature space is established and the features of each kernel function are combined, making it more flexible. In this paper, three kinds of hybrid-kernel SVM models, which combine linear kernel with the polynomial kernel, linear kernel with RBF kernel, polynomial kernel with RBF kernel are established. The hybrid-kernel function LP-SVM is proposed, which combines the linear kernel with the polynomial kernel: 


$$
K_{L P}=\lambda K_{\text {liner }}+(1-\lambda) K_{\text {poly }}
$$

The hybrid-kernel function LR-SVM is proposed, which combines the linear kernel with the RBF kernel:

$$
K_{L R}=\lambda K_{\text {liner }}+(1-\lambda) K_{R B F}
$$

The hybrid-kernel function PR-SVM is proposed, which combines the polynomial kernel with the RBF kernel:

$$
K_{P R}=\lambda K_{p o l y}+(1-\lambda) K_{R B F}
$$

Where $K_{\text {liner }}=x \cdot x_{i}$ is linear kernel function, $K_{p o l y}=\left[\left(x \cdot x_{i}\right)+1\right]^{q}$ is polynomial kernel function, and $K_{R B F}=\exp \left(-\left\|x \cdot x_{i}\right\|^{2} / 2 \sigma^{2}\right)$ is RBF kernel function, and $\lambda$ is the weight coefficient of each two kernel function.

\section{Hybrid-kernel GWO-SVM}

As mentioned above, the construction of a hybrid-kernel SVM model needs to be set up for the regularization parameters $\mathrm{C}$, the kernel function $g$ and the weight coefficient of two kernel functions $(\lambda)$. As a result, the GWO algorithm is used to optimize the three parameters mentioned above. In addition, the optimization takes the minimum error rate of SVM as the fitness value. The hybrid-kernel GWO-SVM model is established and the overall framework of the proposed model is shown in Fig. 4.

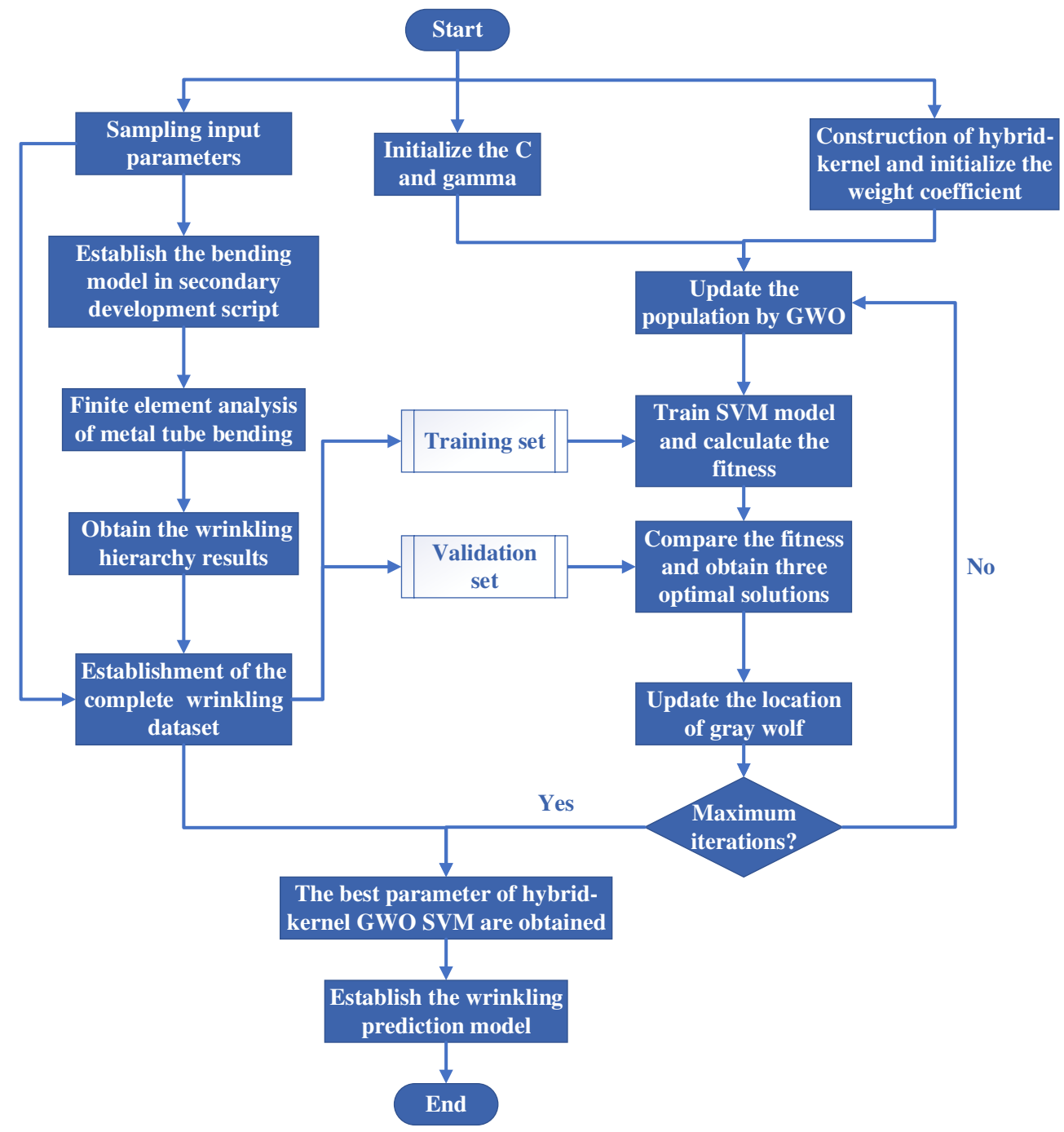

Fig. 4 Flowchart of the predicted model 


\section{Verification and analysis}

\subsection{Case study}

Due to the characteristics of low density and well-performed plasticity, the aluminum alloy tubes are widely utilized as the complex equipment lightweight components. However, the mechanical properties of aluminum tubes are soft so that wrinkling defects are easy to occur in the bending process. Therefore, the aluminum tube is selected as the research object to verify the feasibility of the wrinkle prediction model. The dataset is constructed based on 12 kinds of aluminum alloy tubes, of which the material parameters are shown in Table 1. The 10 different process parameters are sampled by Latin Hypercube sampling as input parameters for the machine learning model, as shown in Table 2. To compare the effect of the number of samples on predicted accuracy, we sampled 120, 240, 360, 480 groups of samples, respectively.

Table 1 Material property

\begin{tabular}{lllllll}
\hline Property & $1060-\mathrm{H} 12$ & $2014-\mathrm{T} 4$ & $2024-\mathrm{O}$ & $2219-\mathrm{T} 81$ & $3003-\mathrm{H} 18$ & $5052-\mathrm{H} 32$ \\
\hline$E(\mathrm{MPa})$ & 69000 & 72400 & 72400 & 72000 & 69000 & 70000 \\
$\sigma_{s 0}(\mathrm{MPa})$ & 75 & 290 & 75 & 350 & 185 & 195 \\
\hline Property & $5052-\mathrm{O}$ & $5454-\mathrm{H} 34$ & $6063-\mathrm{T} 4$ & $7050-\mathrm{T} 7451$ & $6063-\mathrm{T} 6$ & $4032-\mathrm{T} 6$ \\
\hline$E(\mathrm{MPa})$ & 70000 & 70000 & 69000 & 72000 & 69000 & 79000 \\
$\sigma_{s 0}(\mathrm{MPa})$ & 90 & 240 & 90 & 470 & 215 & 315 \\
\hline
\end{tabular}

Table 2 Process parameter and its range

\begin{tabular}{ccccccccccc}
\hline Parameters & $D(\mathrm{~mm})$ & $t / D$ & $R / D$ & $\mu$ & $t_{0}(\mathrm{~mm})$ & $\begin{array}{c}d V p \\
(\mathrm{~mm} / \mathrm{s})\end{array}$ & $\begin{array}{c}L p 0 \\
(\mathrm{~mm})\end{array}$ & $\omega(\mathrm{rad} / \mathrm{s}$ & $L(\mathrm{~mm})$ & $r\left({ }^{\circ}\right)$ \\
\hline Range & $20-200$ & $0.01-$ & $2-5$ & $0.05-$ & $0.1-0.25$ & $-0.25-0.25$ & $20-200$ & $0.5-1.5$ & $200-$ & $30-60$ \\
& & 0.025 & & 0.2 & & & & & 1000 & \\
\hline
\end{tabular}

After the dataset is established, the dynamic analysis is carried out in ABAQUS/Explicit. As shown in Fig. 5, the bending model is consisted with a tube, bending die, clamp die, pressure die, wiper die and insert die. The former one is set as a deformation while others are set as discrete rigid. After the assembling of each parts, the step is divided into initial step and step 1 , where the step time is set. To speed up the simulation, we directly define the mass scaling factors, giving incrementation of time to the dynamic model. Subsequently, the contact between tube surface and die are defined as surface to surface, using penalty contact method, where the contact interaction property is also set. The example of contact settings is shown in fig. 5 . Next, to define the constraint, we fix the section of the tube where the bend starts and the reference point of clamp die together and set wire between the references of clamp die and bending die. In this case, the bending die can drive the clamp die and the tube to rotate synchronously. The boundary conditions are also set include 3 aspects. The bending die is given a rotational angular velocity along the $\mathrm{X}$-axis in step 1 . The wiper die is fixed in both initial step and step 1 . Then, the wiper die is given a linear velocity along the Z-axis in step 1. Finally, we mesh the complex model via the global distribution method, modified the size of the seeds based on the diameter of the tube in each model. For each model, the setting parameters are all set in Python using secondary development script. After finite element analysis, the INP files before and after bending are imported into MATLAB for hierarchical processing analysis. Having built the complete dataset, the prediction model can be set up in LIBSVM. The process of modeling repeats 10 times to eliminate the influence of sample selection. The five kinds of SVM (improved GWO-SVM with three kinds of hybrid kernel function, GWO-SVM and traditional SVM) are applied to data in this paper. 


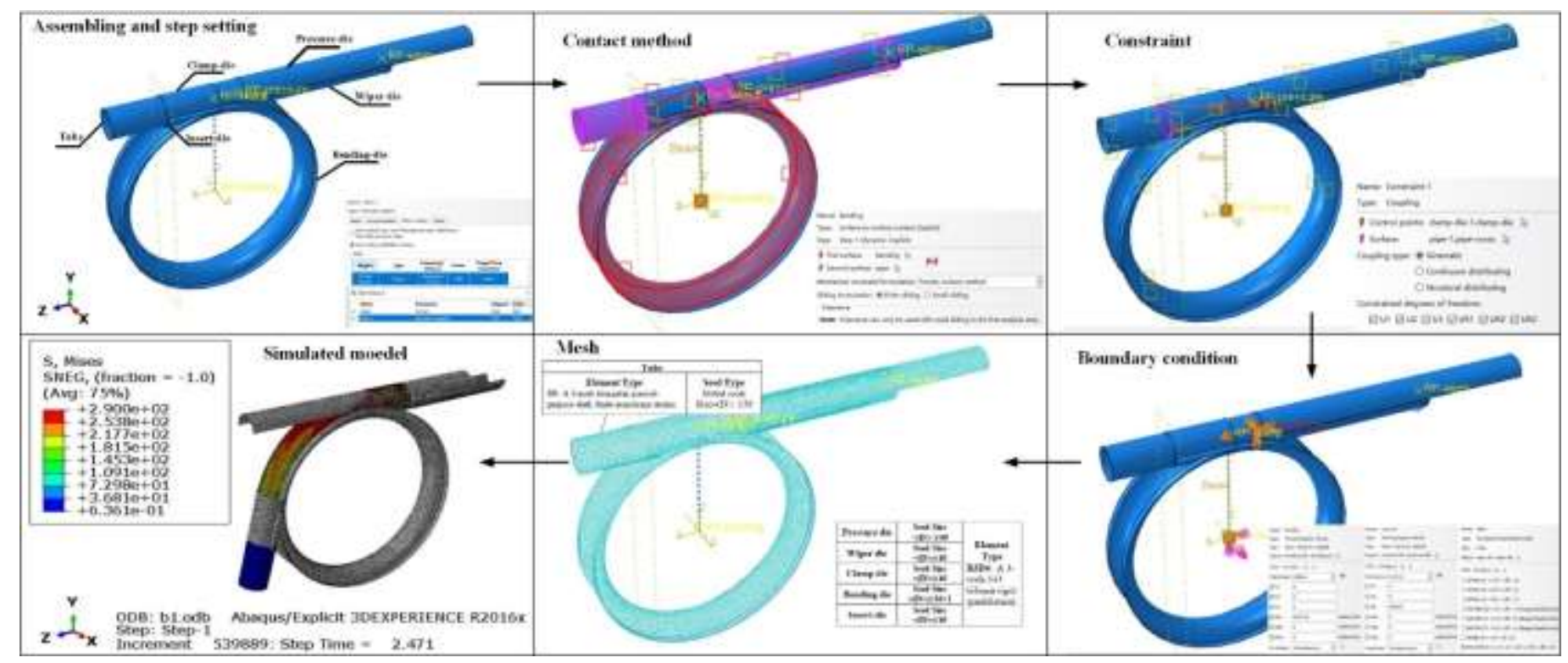

Fig. 5 Simulation setup in ABAQUS/explicit

\subsection{Results and Comparison}

\subsubsection{Experimental results}

To verify the efficiency and accuracy of the proposed method, a 6063 Aluminum Alloy tube is arranged to bend with KMA50-CNC-320 bender, as shown in Fig. 6(a). The tube has a diameter of $27 \mathrm{~mm}$, thickness of $60 \mathrm{~mm}$ and the bending radius is of $60 \mathrm{~mm}$. There are 9 bends as shown in Fig. 6(b). Among them, the angle of 1,7 bends are $75^{\circ}$, while that of other bends are $90^{\circ}$. Apparently, three of the bends $(2,3,7)$ are wrinkled, of which the specific information is measured via a Vernier micrometer. The wrinkle classification method mentioned above is used to classify the bends. We select 3 wrinkling bends and a non-wrinkling bends to verify the reliability of SVM model for predicting wrinkle classification. The process parameters and material parameters of the bent tube are taken as prediction set. The improved LR_GWO_SVM model is used to predict the wrinkle classification. The predicted results are in an agreement with the measured results, which further illustrates the feasibility of the method. The results show that it could realize the hierarchical prediction of tube wrinkling with known parameters. Experimental diagram and measuring method are shown in Fig. 6 and the measuring results and prediction results are shown in Table 3.

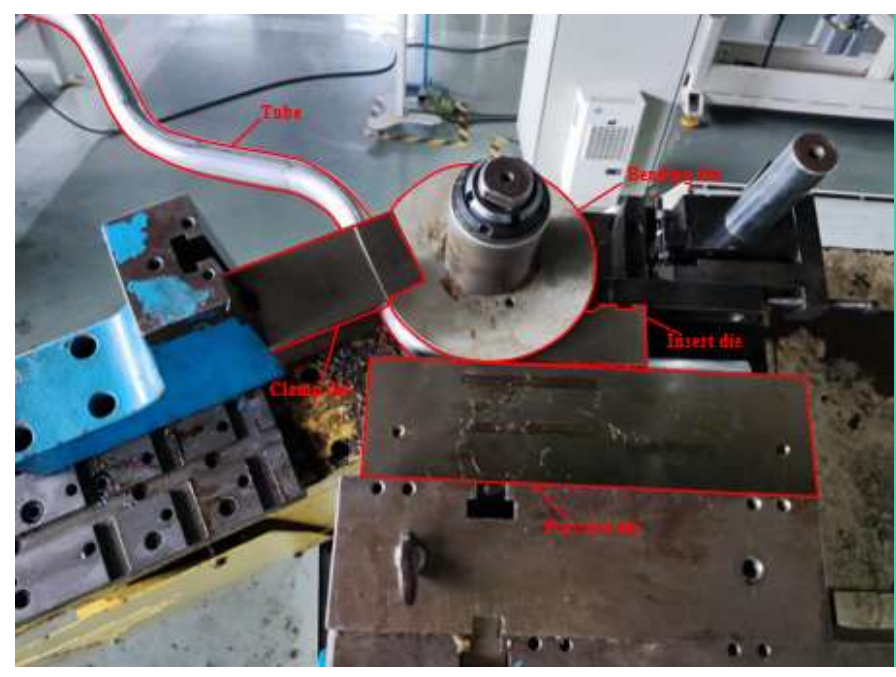

(a)

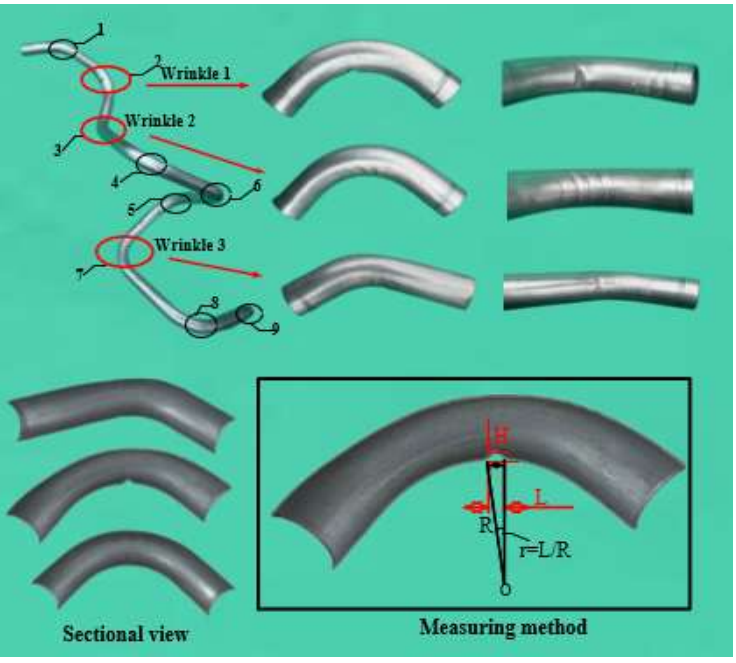

(b)

Fig. 6 Bending equipment (a) and experimental wrinkling measurement (b)

Table 3 Comparison of experimental data and prediction result

\begin{tabular}{|c|c|c|c|c|c|c|}
\hline \multicolumn{3}{|c|}{ Measured value } & \multicolumn{4}{|c|}{ Calculated value } \\
\hline$H / D$ & Length between troughs $(L)$ & $n$ & $J / D$ & $P$ & Actual Category & Predict category \\
\hline
\end{tabular}




\begin{tabular}{cccc|cccc}
\hline 1 & 2.667 & 5.08 & 1 & 4.855 & 0.0203 & 2 & 2 \\
\hline 2 & 1.060 & 4.647 & 1 & 4.441 & 0.009 & 1 & 1 \\
\hline & 0.660 & 3.513 & & 3.357 & 0.007 & & 3 \\
3 & 0.453 & 5.240 & 3 & 5.008 & 0.003 & 3 & \\
& 0.660 & 2.793 & & 2.669 & 0.009 & & 0 \\
\hline 4 & 0 & 0 & 0 & 0 & - & 0 & \\
\hline
\end{tabular}

\subsubsection{Comparison with different algorithms}

The comparison test algorithm is coded in MATLAB2021a, running on a LAPTOP (CPU: intel core i5-7200U,2.50GHz,2.71 $\mathrm{GHz}, 8 \mathrm{~GB}$ ) for ten times to compare the accuracy of the SVM algorithms with different hybrid-kernel functions. The most accurate SVM algorithm is selected for wrinkling prediction. As the results are shown in Fig. 7, Liner kernel function combined with RBF kernel function (LR_SVM_GWO) has the highest accuracy for 8 times of ten runs. The average accuracy for ten runs is $74.5 \%$ and the highest accuracy in ten runs is $83.0 \%$. This indicates the higher prediction ability of LR_SVM_GWO. However, LP_SVM_GWO, which uses the hybrid kernel function of liner kernel and polynomial kernel, has lower accuracy. The average accuracy of LP_SVM_GWO is 70.0\%, while the average of RBF kernel function combined with polynomial kernel function is $40.0 \%$. As a result, LR_SVM_GWO was selected as the improved SVM algorithm to further verify the feasibility of wrinkle prediction model in this paper. Apparently, the prediction accuracy of the LR_GWOSVM approach exceeds GWO-SVM and traditional SVM. Among the ten runs, the LR_GWO_SVM has the highest average accuracy of $74.5 \%$, which is higher than the accuracy of GWO-SVM $71.0 \%$ and traditional SVM $63.8 \%$. The results show the superiority of the improved GWO-SVM model and its reliability in predicting the wrinkling of metal tube bending.

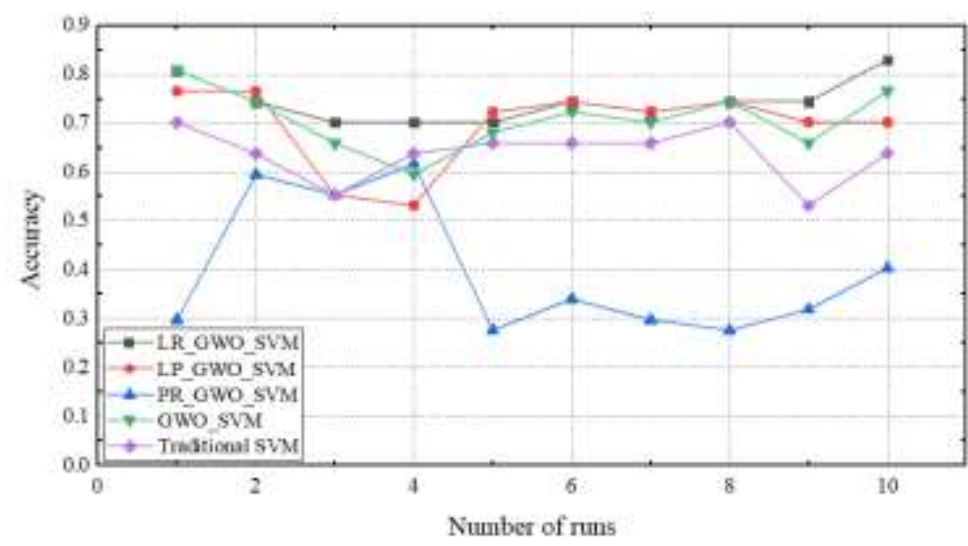

Fig. 7 Comparison of prediction accuracy for different SVM models

The real categories and predicted results of LR_GWO_SVM, GWO_SVM and traditional SVM is shown in Fig. 8. From the calculation results, we can see that higher prediction accuracy is achieved by the improved method. To further evaluate the superiority of the improved method, three statistical indices are utilized to measure the forecasting accuracy. These indices are the mean absolute error (MAE), mean square error (MSE) and the root mean square error (RMSE) [Error! Reference source not found.]. The values can reflect the difference between the virtual data and the predict data which are often as criterion of evaluating machine learning models. The smaller values indicate higher forecast performance. These indices are defined in Eq. (14), where $y_{n}$ is the real value and $\boldsymbol{y}_{n}^{\mathbf{l}}$ is the predicted value.

$$
\begin{aligned}
& M S E=\frac{1}{m} \sum_{i=1}^{m}\left(y_{i}-y_{i}\right)^{2} \\
& M A E=\frac{1}{m} \sum_{i=1}^{m}\left|y_{i}-y_{i}\right| \\
& R M S E=\sqrt{\frac{1}{m} \sum_{i=1}^{m}\left(y_{i}-y_{i}\right)^{2}}
\end{aligned}
$$

As it can be seen from Fig. 9, the three error values of LR_GWO_SVM are the smallest of the five models with the MAE of 0.445 , the MSE value of 1.01 and the RMSE value of 0.992 , which indicates that the improved prediction model is more 
reliable. Compared with the contrast models, prediction results have been improved successfully.

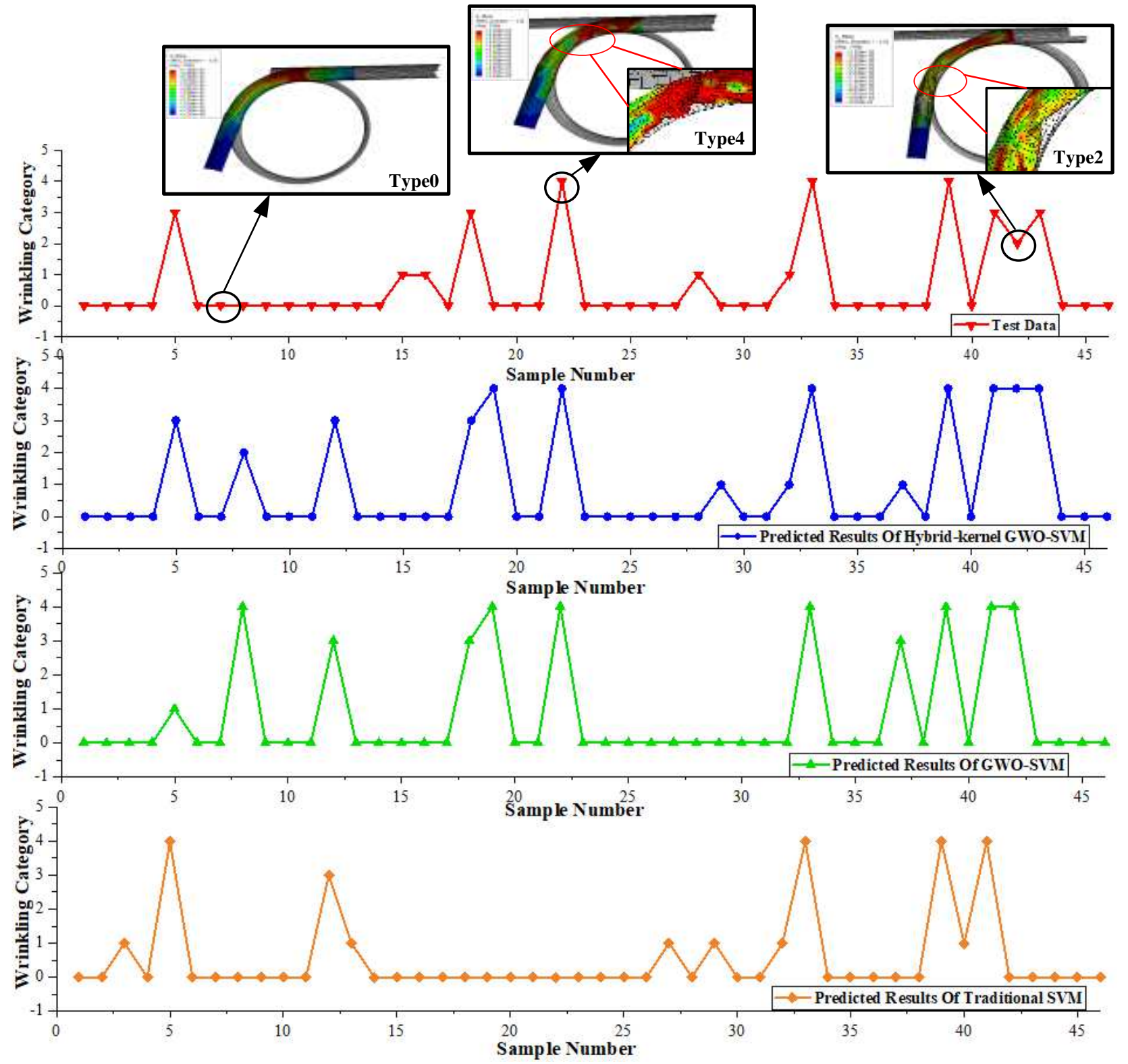

Fig. 8 Real categories and predicted results of four methods (real data-+, results of LR_GWO_SVM-O, results of GWO_SVM- $\triangle$, results of traditional SVM- $\diamond)$

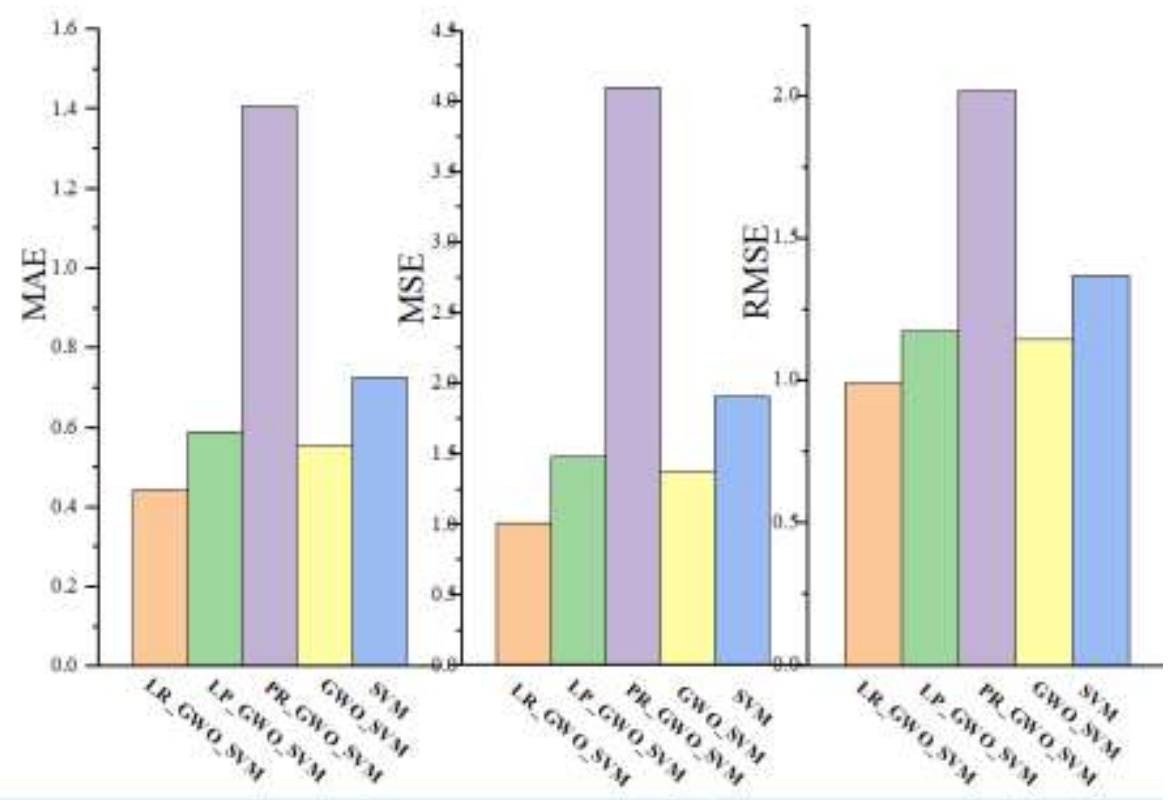




\subsection{Analysis and discussion}

\subsubsection{The influence of training sample amount on prediction accuracy}

Eliminating the wrong samples, there are 118, 236, 355 and 473 groups of samples left to apply the prediction model. As shown in Fig. 10, with the increase of the number of samples, the accuracy of the prediction model established by the four algorithms has been improved to some extent. When the sample size was increased to 473, the accuracy of LR_GWO_SVM increased from $64.3 \%$ to $75.9 \%$ comparing with 118 samples, increased by $18.0 \%$. The accuracy of GWO_SVM and traditional SVM was also increased from $60.4 \%$ to $75.2 \%$ and $59.8 \%$ to $69.5 \%$. The larger the sample size, the more comprehensive the training data, the machine learning model will show better performance. At the same time, if the number of samples is too small, it is difficult to get dependable prediction model. Therefore, in the application, getting as much samples as possible is a way to continuously improve the prediction model and achieve higher accuracy.

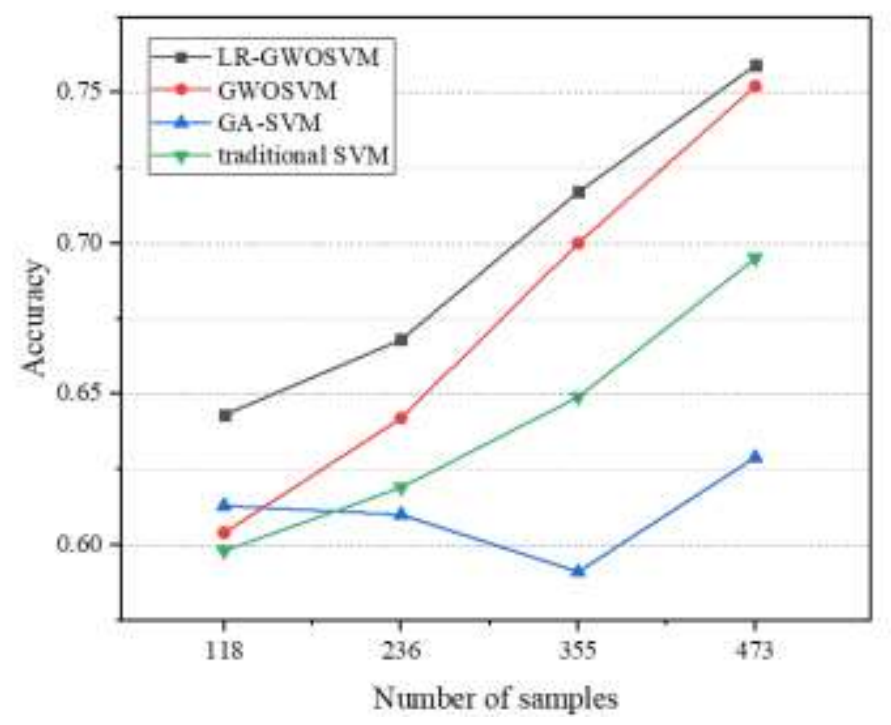

Fig. 10 Effect of samples amount on prediction accuracy

\subsubsection{The influence of bending process parameters on wrinkling}

It is of great importance to analyze the effect of process parameters on wrinkling in metal tube bending, especially that of $R / D$ and $t / D$. It is because that both the relative bending radius and the relative wall thickness have great impact on the tangential compressive stress on the concave side of the tube wall, which will seriously influence the wrinkling tendency of the inside wall. As shown in Fig. 11, the bending of metal tubes with relative wall thickness of 0.008-0.025 and relative bending radius of 2-5 is made by simulation model (a) the newly prediction model (b). The influence of other variables on wrinkling results can be limited by the control variable method. The influence tendency of relative bending radius and wall thickness on the wrinkling can be analyzed from the results. As can be seen from the diagram, the smaller the $t / D$, the higher the wrinkling degree. Similarly, the smaller the $R / D$, the higher the hierarchy of wrinkling. That's because low relative wall thickness and bending radius both lead to unequal deformation, which will result in more wrinkling tendency. The prediction results are consistent with the simulation results and theoretical analysis, which further proves the reliability of the prediction model. 


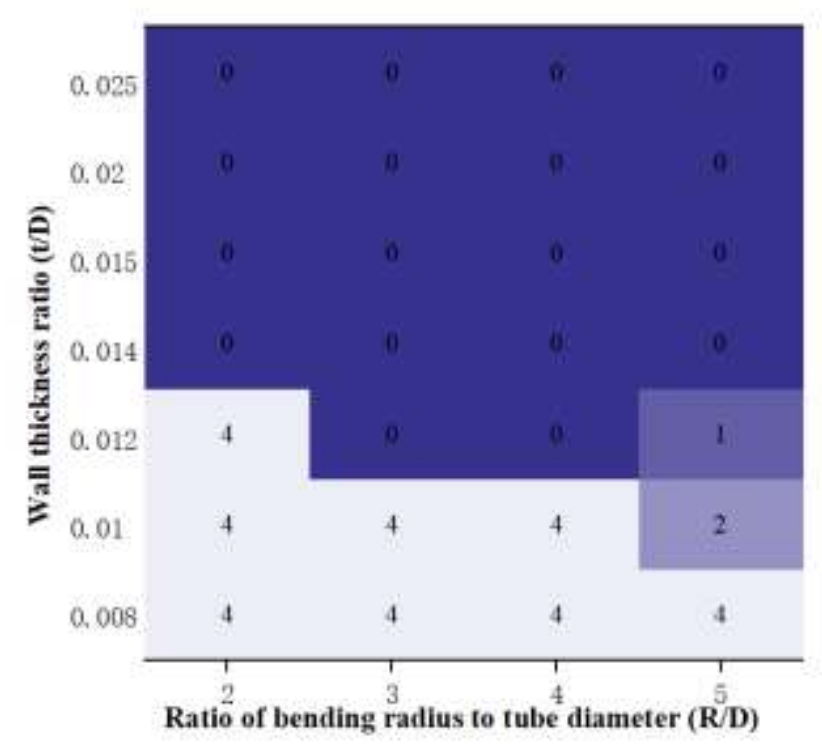

(a) Experiment results

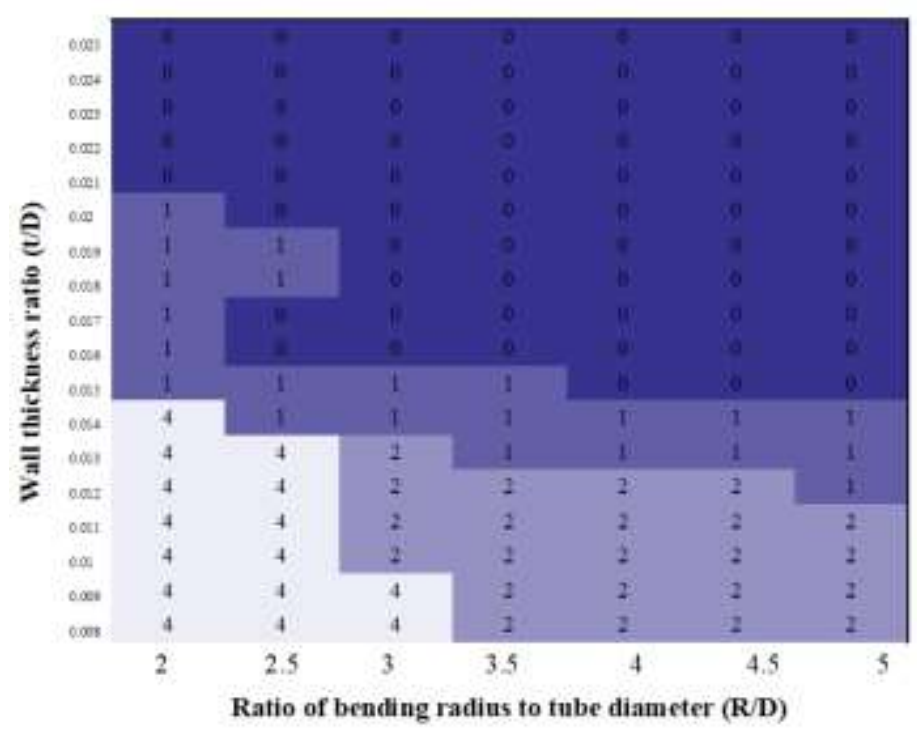

(b) Prediction results

Fig. 11 Effect of the ratio of bending radius to tube diameter $(R / D)$ and wall thickness ratio $(t / d)$

\section{Conclusion}

Bending defect is a general phenomenon that may occur in metal tube forming. Among all defects, the bending-inside wrinkling caused by the non-uniform compressive stress is a zero-tolerated defect. Therefore, realizing the prediction of bending wrinkling can make a significant contribution to bending quality. Given that the current wrinkling detection approach, suffering from the lack of insight into wrinkling mechanism, is normally posteriori, we proposed a hierarchical prediction method for metal tube bending wrinkling detection to obtain the priori wrinkling condition for a certain go-to-bend tube. Based on the good classification performance of SVM, it is presented for the prediction of wrinkling models. Due to the great influence of SVM parameters and kernel function on the prediction accuracy, GWO and hybrid kernel method are integrated to optimize the prediction model and achieve better prediction ability.

A series of aluminum alloy tubes is chosen as the case study of the proposed method. The aluminum alloy tubes bending wrinkling database, which consists of 12 kinds of aluminum tubes considering 12 parameters in metal tube bending process, is constructed. To train and obtain the wrinkling prediction model, five SVM-based algorithms, viz., GWO-SVM with three kinds of hybrid kernel, GWO-SVM, and traditional SVM, are compared. The result of LR-GWO-SVM shows the best performance of $74.5 \%$ average accuracy, which is higher than that of GWO-SVM (70.9\%) and that of traditional SVM (63.8\%). Furthermore, an experiment based on the 6063-aluminum tube bending is carried out. The results show that the novel hybrid-kernel GWO-SVM method is a proper model for hierarchically predicting metal tube bending wrinkling detection.

\section{Acknowledgements}

This work has been funded by the Joint Funds of the National Natural Science Foundation of China (U20A20287), the National Natural Science Foundation of China (51905476), the Zhejiang Province Public Welfare Technology Application Research Project, China (LGG22E050008 and GG22E057584).

\section{Competing Interests}

Authors declare that they have no conflict of interest.

\section{Ethical Approval}

No applicable

\section{Consent to Participate}

No applicable 


\section{Consent to Publish}

Authors consent to publish this article

\section{Reference}

1. Li H, Yang H, Zhang ZY, Wang ZK (2013) 'Size effect' related bending formability of thin-walled aluminum alloy tube. Chinse J Aeronaut 26: 230-241

2. Li H, Yang H, Zhan M, Gu RJ (2006) Forming characteristics of thin-walled tube bending process with small bending radius. T Nongerr Metal Soc 16: 613-623

3. Wang Z, Lin Y, Qiu L, Zhang S, Fang D, He C, Wang L (2021) Spatial variable curvature metallic tube bending springback numerical approximation prediction and compensation method considering cross-section distortion defect. Int J Adv Manuf Technol. https://doi.org/10.1007/s00170-021-08051-w

4. Yue YB, Yang H, Zhan M, Kou YL, Li H (2007) Experimental study on thinning of thin-walled tube NC bending process with small bending radius Experimental study on thinning of thin-walled tube $\mathrm{NC}$ bending process with small bending radius. Forging \& Stamping Technol 32: 58-62

5. Munz D, Mattheck C (1982) Cross-Sectional Flattening of Pipes Subjected To Bending. Int J Pres Ves Pip 10: 421-429

6. He Y, Lin Y (2004) Wrinkling analysis for forming limit of tube bending processes. J Mater Process Tech 152: 363-369

7. Li H, Yang H, Zhan M, Sun ZC, Gu RJ (2007) Role of mandrel in NC precision bending process of thin-walled tube. Int J Mach Tool Manu 47: 1164-1175

8. Zhang S, Fu M, Wang Z, Fang D, Lin W, Zhou H (2021) Springback prediction model and its compensation method for the variable curvature metal tube bending forming. Int J Adv Manuf Technol 112: 3151-3165

9. Liu TJ, Wang YJ, Wu JJ, Xia XJ, Wang W, Wang SH (2014) Springback of extruded 2196-T8511 and 2099-T83 Al-Li alloys in stretch bending, in 11th International Conference on Technology of Plasticity. Elsevier Science Bv, Amsterdam, pp 981-986

10. Li C, Yang H, Zhan M, Xu XD, Li GJ (2009) Effects of process parameters on numerical control bending process for large diameter thin-walled aluminum alloy tubes. T Nonferr Metal Soc 19: 668-673

11. Huang T, Yang F, Zhan M et al (2018) Section Flattening in Numerical Control Bending Process of TA18 High Strength Tube. Rare Metal Mat Eng 47: 2347-2352

12. Zhao C, Han Z, Du B, Zhang X, Xie J (2019) Wrinkling prediction of aluminum alloy tubes during reduced diameter compression forming. Int J Adv Manuf Technol 106: 65-75

13. Yang H, Yan J, Zhan M, Li H, Kou YL (2009) 3D numerical study on wrinkling characteristics in NC bending of aluminum alloy thin-walled tubes with large diameters under multi-die constraints. Comp Mater Sci 45: 1052-1067

14. Yang H, Li H, Zhan M (2010) Friction role in bending behaviors of thin-walled tube in rotary-draw-bending under small bending radii. J Mater Process Tech 210: 2273-2284

15. Cui XL, Wang XS, Yuan SJ (2018) Effects of mechanical property parameters on wrinkling behavior of thin-walled tubes in hydroforming process. Int J Adv Manuf Technol 100: 729-740

16. Hasanpour K, Barati M, Amini B, Poursina M (2013) The effect of anisotropy on wrinkling of tube under rotary draw bending. J Mech Sci Technol 27: 783-792

17. Alexander C, Kulkarni S, Asme (2009) Evaluating The Effects Of Wrinkle Bends On Pipeline Integrity. In: Proceedings of the Asme International Pipeline Conference, Amer Soc Mechanical Engineers, New York, pp 61-74

18. Li H, Yang H, Zhan M, Gu RJ (2006) A new method to accurately obtain wrinkling limit diagram in NC bending process of thin-walled tube with large diameter under different loading paths. J Mater Process Tech 177: 192-196

19. Dib MA, Oliveira NJ, Marques AE, Oliveira MC, Fernandes JV, Ribeiro BM, Prates PA (2019) Single and ensemble classifiers for defect prediction in sheet metal forming under variability. Neural Comput \& Applic 32: 12335-12349

20. Inamdar MV, Date PP, Desai UB (2000) Studies on the prediction of springback in air vee bending of metallic sheets using an artificial neural network. J Mater Process Tech 108: 45-54

21. Gisario A, Barletta M, Conti C, Guarino S (2011) Springback control in sheet metal bending by laser-assisted bending: Experimental analysis, empirical and neural network modelling. Opt Laser Eng 49: 1372-1383

22. Tavara S (2019) Parallel Computing of Support Vector Machines. ACM Comput Surv 51: 1-38

23. Thanh CL, Trong NN, Khatir S, Phuoc TN, Mirjalili S, Nguyen KD (2021) An efficient approach for damage identification based on improved machine learning using PSO-SVM. Eng Comput-Germany. https://doi.org/10.1007/s00366-021-01299-6 
24. Phan, A.V., Nguyen, M.L., Bui, L.T. (2017) Feature weighting and SVM parameters optimization based on genetic algorithms for classification problems. Appl Intell 46: 455-469. https://doi.org/10.1007/s10489-016-0843-6

25. Mirjalili S, Mirjalili SM, Lewis A (2014) Grey Wolf Optimizer. Adv Eng Softw 69: 46-61

26. Shi Q, Zhang H (2021) Fault Diagnosis of an Autonomous Vehicle With an Improved SVM Algorithm Subject to Unbalanced Datasets. Ieee T Ind Electron 68: 6248-6256

27. Xu RR, Lan HJ (2020) Demand Forecasting Model of Aquatic Cold Chain Logistics Based on GWO-SVM. In: Conference Proceedings of the 8th International Symposium on Project Management, Wuhan, China, pp:1088-1098

28. Tian YJ, Shi Y, Liu XH (2012) Recent Advances On Support Vector Machines Research. Technol Econ Dev Eco 18: 5-33

29. Wang H, Li J, Yang F (2014) Overview of support vector machine analysis and algorithm. Application Research of Computers 31: 1281-1286

30. Evgeniou T, Evgeniou M (2001) Support Vector Machines: Theory and Applications. LNCS 302: 249-257

31. Xuzhou, Science CJC (2011) Advances of Support Vector Machines(SVM) Comp Sci 38:14-17

32. Zhou J, Huang S, Wang M, Qiu Y (2021) Performance evaluation of hybrid GA-SVM and GWO-SVM models to predict earthquake-induced liquefaction potential of soil: a multi-dataset investigation. Eng Comput-Germany. https://doi.org/10.1007/s00366-021-01418-3

33. Zhou Y, Xiao W, Liu M, Zhang Y, Wang Y (2018) Hybrid Kernel LSSVM Model for Prediction of Short-Term Wind Speed. In: ICCC2018 IEEE 4th International Conference on Computer and Communications, Chengdu, China, pp: 2641-2644

34. Gupta, D. (2017) Training primal K-nearest neighbor based weighted twin support vector regression via unconstrained convex minimization. Appl Intell 47: 962-991. https://doi.org/10.1007/s10489-017-0913-4 